\title{
Efectividad de las tecnologías de la información y comunicación en la adherencia terapéutica de pacientes con Hipertensión Arterial y Diabetes Mellitus
}

\author{
Diana Patricia González-Ruiz, Daniela Alejandra Getial-Mora, María Alejandra Higidio-Miranda, Sandra Milena \\ Hernández-Zambrano
}

Facultad de Enfermería. Fundación Universitaria de Ciencias de la Salud. Colombia

Como citar este artículo:

González-Ruiz DP, Getial-Mora DA, Higidio-Miranda MA, Hernández-Zambrano SM. Efectividad de las tecnologías de la información y comunicación en la adherencia terapéutica de pacientes con Hipertensión Arterial y Diabetes Mellitus.

Enferm Nefrol. 2020 Ene-Mar;23(1):22-32

\section{Resumen}

Introducción: La hipertensión arterial y la diabetes mellitus son dos de las principales causas de morbimortalidad, siendo las precursoras de la enfermedad renal crónica, catalogándose como un problema de salud pública que afecta a uno de cada diez adultos en el mundo. La falta de cumplimiento al tratamiento es la mayor causa de fracaso en los programas de promoción y prevención, con esta revisión sistemática se busca aportar a los profesionales de enfermería evidencia científica a través del uso de las tecnologías de la información y la comunicación que permitan mejorar la adherencia terapéutica.

Objetivo: Identificar la efectividad de las tecnologías de la información y la comunicación en la adherencia terapéutica en personas con Hipertensión Arterial y Diabetes Mellitus.

Material y Método. Se realizó una revisión sistemática de literatura en las bases de datos CUIDEN, CINAHL, COCHRANE, LILACS, PUBMED y OVID NURSING. Después de aplicar filtros de selección y listas de verificacion de calidad metodológica se obtuvieron 18 artículos para análisis, 12 ensayos clínicos aleatorizados, 3 estudios cuasi-experimentales y 3 revisiones sistemáticas. Se

\section{Correspondencia:}

Diana Patricia González Ruiz

E-mail: dpgonzalez@fucsalud.edu.co clasificó el nivel de evidencia de los estudios según el instituto Joanna Briggs.

Resultados. Se establecieron 3 categorías: Intervenciones unicomponente, intervenciones multicomponente y rol de enfermería en el uso de TICs para favorecer la adherencia.

Conclusiones. Dentro del cuidado de la salud actualmente hay hallazgos iniciales que apoyan la efectividad del uso de las tecnologías de la información y la comunicación convirtiéndola en una herramienta que favorece la adherencia al tratamiento en pacientes con hipertensión arterial y diabetes mellitus.

PALABRAS CLAVE: cumplimiento y adherencia al tratamiento; cumplimiento del paciente; tecnología de la información; ciencia de la información; hipertensión arterial; diabetes mellitus.

\section{Effectiveness of information and communication technologies in therapeutic adherence of patients with Arterial Hypertension and Diabetes Mellitus}

\section{Abstract}

Introduction: Arterial hypertension and diabetes mellitus are two of the main causes of morbidity and mortality, being the precursors of chronic kidney disease 
and classified as a public health problem that affects one in ten adults in the world. Lack of compliance with treatment is the major cause of failure in promotion programs and prevention. This systematic review seeks to provide scientific evidence to nursing professionals using information and communication technologies that improve therapeutic adherence.

Objective: Identify the effectiveness of information and communication technologies in therapeutic adherence in people with Arterial Hypertension and Diabetes Mellitus.

Material y Method: A systematic literature review was carried out in the CUIDEN, CINAHL, COCHRANE, LILACS, PUBMED and OVID NURSING databases. After applying the selection criteria and methodological quality checklists, 18 articles were obtained for analysis: 12 randomized clinical trials, 3 quasi-experimental studies and 3 systematic reviews. The level of evidence of the studies was classified according to the Joanna Briggs Institute.

Results: Three categories were established: single interventions, multicomponent interventions and nursing role in the use of ICTs to favour adherence.

Conclusions: Currently, within health care, there are initial findings that support the effectiveness of the use of information and communication technologies, being a tool that favours therapeutic adherence in patients with arterial hypertension and diabetes mellitus.

KEYWORDS: Compliance and therapeutic adherence; patient compliance; information technology; information science; hypertension; diabetes mellitus.

\section{Introducción}

La hipertensión arterial y la diabetes mellitus son las principales causas de morbimortalidad en el mundo ${ }^{1-3}$. La organización mundial de la salud (OMS) sitúa las enfermedades cardiovasculares y la diabetes mellitus dentro de las primeras cinco causas de mortalidad en adultos de ambos sexos proyectada para el 2030 como el motivo de más de 8 millones de muertes a partir de los 30 años ${ }^{4}$, siendo a su vez factores de riesgo para el desarrollo de enfermedad renal crónica (ERC) catalogándola como un problema de salud pública que afecta a uno de cada diez adultos en el mundo 5 .
El término adherencia se define como "el cumplimiento de los tratamientos farmacológicos, como el seguimiento de las recomendaciones higiénico-dietéticas y la adopción de cambios de estilos de vida en los pacientes ${ }^{\prime \prime}$. Este es uno de los principales aspectos para lograr el control de la enfermedad crónica, reducir la tasa de complicaciones y prolongar la supervivencia. La OMS, reveló que en los países desarrollados la adherencia entre los pacientes con enfermedades crónicas no es mayor del $50 \%{ }^{7}$, la falta de cumplimiento al tratamiento es la principal causa de fracaso en los programas de promoción y prevención, por lo tanto, se convierte en un desafío constante encontrar formas eficaces de apoyo al tratamiento ${ }^{8}$. Por lo anterior, en los servicios sanitarios se ha implementado el uso de las tecnologías de la información y la comunicación (TICs), por parte de los profesionales de enfermería para apoyar el seguimiento y educación a los pacientes de forma participativa, así como coordinar su cuidado a distancia 9,10.

Las TICs entendidas como el conjunto de tecnologías que van a permitir la producción, almacenamiento, registro y presentación de la información, en forma de señal acústica, óptica o electromagnética ${ }^{11}$, se encuentran presentes en las entidades de salud, que buscan aumentar la eficacia de los tratamientos y la calidad de vida de los pacientes ${ }^{12,13}$. Teniendo en cuenta el bajo grado de adherencia terapéutica en personas con diabetes mellitus e hipertensión arterial, surge la motivación de indagar frente a la efectividad del uso de las TICs para lograr la adherencia terapéutica ${ }^{14}$.

Con esta revisión sistemática se busca aportar a los profesionales de enfermería evidencia científica para el desarrollo de estrategias y herramientas educativas y de intervención que permitan mejorar la adherencia terapéutica en los pacientes desde la promoción de la salud, la prevención de la enfermedad y el autocuidado de la misma, a través del uso de las TICs (dispositivos móviles, mensajes de texto, Ilamadas, correos electrónicos con recordatorios de toma de medicamento o educación continua para el abordaje de la enfermedad). Lo anterior con el fin de disminuir la morbi-mortalidad por enfermedades como la diabetes y la hipertensión, y limitar las predisposiciones a la enfermedad renal. Por tanto, el objetivo de esta revisión es identificar la efectividad de las TICs en la adherencia terapéutica en personas con hipertensión arterial y diabetes mellitus. 


\section{Material y Método}

Se efectuó una revisión sistemática durante el primer semestre del 2018, entendida como "una síntesis de evidencias, que maneja un riguroso proceso que identifica, evalúa y sintetiza estudios que van a dar respuesta a una pregunta clínica específica y extraer conclusiones sobre los datos recopilados, esta analiza de forma sistematizada la efectividad de las intervenciones de modo que responde fundamentalmente a la pregunta planteada"15.

Para dar respuesta a la pregunta de investigación se realizó una búsqueda de artículos en las siguientes bases de datos: CUIDEN, CINAHL, COCHRANE, LILACS, PUBMED y OVID NURSING. Los criterios de elegibilidad de los estudios fueron: Revisiones sistemáticas, estudios cuasi-experimentales, ensayos clínico aleatorizados, publicados entre 2010 y 2018, en los idiomas español, inglés, portugués.

Se utilizaron palabras clave traducidas a encabezamientos de materia médica (MeSH) y descriptores en ciencias de la salud (DeCS) formulando la siguiente ecuación de búsqueda: "Treatment Adherence and Compliance" OR "Patient Compliance" AND "Information Technology" OR "Information Science" AND "Hypertension" OR "Diabetes Mellitus".

Tras aplicar la estrategia de búsqueda se encontraron 7.525 artículos. Después de aplicar criterios de inclusión y de emplear listas de verificación de calidad metodológica, se obtuvieron 18 artículos para análisis: 12 ensayos clínicos aleatorizados, 3 estudios cuasi-experimentales, 3 revisiones sistemáticas. El diagrama de flujo de la información se muestra en la Figura 1. EI texto completo de los documentos incluidos se obtuvo por la biblioteca virtual de la Fundación Universitaria de Ciencias de la Salud.
Se aplicó el proceso de lectura crítica a los 18 estudios seleccionados, utilizando como instrumento de valoración la lista de comprobación CONSORT para ensayos clínicos aleatorizados, TREND para estudios cuasi-experimentales y AMSTAR para revisiones sistemáticas. Se utilizó la propuesta del Instituto Joanna Briggs para asignación de grado de evidencia y fuerza de recomendación.

Para el análisis de resultados, se diseñó una matriz en el programa de Microsoft Office Excel, donde cada in- 
vestigador la diligenció según: temas de relevancia para cada artículo, agrupación de los temas en común para construcción de categorías e inclusión de proposiciones, posterior a esto se realizó la triangulación entre los investigadores de los resultados individuales que arrojaron 3 categorías de análisis.

Se tuvieron en cuenta las consideraciones éticas de la resolución 8430 de 1993, para estudios documentales en donde se establece que es una investigación sin riesgo y la ley 44 de 1993 donde se esclarece la responsabilidad de los investigadores con relación de la protección al derecho de autor de los artículos utilizados en este documento.

\section{Resultados}

Se obtuvieron 18 artículos para lectura y análisis correspondientes al $66,6 \%$ de estudios controlados aleatorizados, $16,6 \%$ de estudios cuasi-experimentales y $16,6 \%$ de revisiones sistemáticas. El país que más reporta investigación en esta temática es Estados Unidos con 10 artículos; respecto al idioma se evidencia mayor publicación en inglés. Los años reportados de los artículos oscilan entre los años 2010-2018. La muestra agrupada de los 18 estudios incluidos fue de 24.058 individuos con hipertensión arterial y diabetes mellitus; la edad de rango de participantes fue de 18 a 89 años (ver Tabla 1).

Tabla 1. Artículos seleccionados.

\begin{tabular}{|c|c|c|c|c|}
\hline $\begin{array}{l}\text { Referencia } \\
\text { bibliográfica }\end{array}$ & Idioma, País & Objetivo & $\begin{array}{l}\text { Diseño/metodológico } \\
\text { Nivel de evidencia }\end{array}$ & Participantes \\
\hline $\begin{array}{l}\text { Canata et al., } \\
\text { (2017). }\end{array}$ & $\begin{array}{l}\text { Portugués. } \\
\text { Brasil. }\end{array}$ & $\begin{array}{l}\text { Evaluar la efectividad del soporte telefónico en } \\
\text { el control metabólico de ancianos con diabetes } \\
\text { mellitus. }\end{array}$ & $\begin{array}{l}\text { Ensayo clínico aleatorizado. } \\
\text { Nivel de evidencia: } 1 c \text {. }\end{array}$ & 63 \\
\hline $\begin{array}{l}\text { Kanadli et al., } \\
(2016) .\end{array}$ & $\begin{array}{l}\text { Inglés. } \\
\text { Turquía. }\end{array}$ & $\begin{array}{l}\text { Investigar el impacto de la educación y el } \\
\text { seguimiento telefónico sobre el autocuidado y el } \\
\text { control metabólico en pacientes diabéticos. }\end{array}$ & $\begin{array}{l}\text { Ensayo clínico aleatorizado. } \\
\text { Nivel de evidencia: } 1 c \text {. }\end{array}$ & 88 \\
\hline $\begin{array}{l}\text { Harrison et al., } \\
\text { (2013). }\end{array}$ & $\begin{array}{l}\text { Inglés. } \\
\text { Estados Unidos. }\end{array}$ & $\begin{array}{l}\text { Evaluar la efectividad de un programa de alcance } \\
\text { telefónico para mejorar el control de la presión } \\
\text { arterial (PA) entre pacientes con hipertensión. }\end{array}$ & $\begin{array}{l}\text { Ensayo clínico aleatorizado. } \\
\text { Nivel de evidencia: } 1 c \text {. }\end{array}$ & 64.773 \\
\hline $\begin{array}{l}\text { Glasgow et al., } \\
\text { (2012). }\end{array}$ & $\begin{array}{l}\text { Inglés. } \\
\text { Estados Unidos. }\end{array}$ & $\begin{array}{l}\text { Determinar si los programas basados en Internet } \\
\text { ofrecen un potencial para la autogestión práctica } \\
\text { y rentable de los programas de enfermedades } \\
\text { crónicas. }\end{array}$ & $\begin{array}{l}\text { Ensayo clínico aleatorizado. } \\
\text { Nivel de evidencia: } 1 c \text {. }\end{array}$ & 358 \\
\hline $\begin{array}{l}\text { Avdal et al., } \\
\text { (2011). }\end{array}$ & $\begin{array}{l}\text { Inglés. } \\
\text { Turquía. }\end{array}$ & $\begin{array}{l}\text { Probar los efectos de proporcionar educación } \\
\text { sobre la diabetes basada en la web a las personas } \\
\text { con diabetes tipo } 2 \text { en el nivel Alc y la asistencia } \\
\text { sanitaria. }\end{array}$ & $\begin{array}{l}\text { Ensayo clínico aleatorizado. } \\
\text { Nivel de evidencia: } 1 c \text {. }\end{array}$ & 105 \\
\hline $\begin{array}{l}\text { Magid et al., } \\
\text { (2013). }\end{array}$ & $\begin{array}{l}\text { Inglés. } \\
\text { Estados Unidos. }\end{array}$ & $\begin{array}{l}\text { Evaluar la efectividad de una intervención de } \\
\text { HBPM con tecnología Heart360 de la Asociación } \\
\text { Estadounidense del Corazón, liderada por un } \\
\text { farmacéutico, en comparación con la atención } \\
\text { habitual (CU) para pacientes con hipertensión no } \\
\text { controlada. }\end{array}$ & $\begin{array}{l}\text { Ensayo clínico aleatorizado. } \\
\text { Nivel de evidencia: } 1 c \text {. }\end{array}$ & 326 \\
\hline $\begin{array}{l}\text { Lee et al., } \\
\text { (2016). }\end{array}$ & $\begin{array}{l}\text { Inglés. } \\
\text { China. }\end{array}$ & $\begin{array}{l}\text { Evaluar si el sistema de BP en la nube integrado } \\
\text { con la entrada de orden médico computarizado } \\
\text { (CPOE) puede mejorar el manejo de la PA en } \\
\text { comparación con la atención tradicional. }\end{array}$ & $\begin{array}{l}\text { Ensayo clínico aleatorizado. } \\
\text { Nivel de evidencia: } 1 c \text {. }\end{array}$ & 218 \\
\hline $\begin{array}{l}\text { Migneault et } \\
\text { al.,(2012). }\end{array}$ & $\begin{array}{l}\text { Inglés. } \\
\text { Estados Unidos. }\end{array}$ & $\begin{array}{l}\text { Evaluar un sistema telefónico automatizado } \\
\text { adaptado culturalmente para ayudar a los adultos } \\
\text { afroamericanos urbanos hipertensos a mejorar } \\
\text { su adherencia a su régimen de medicamentos } \\
\text { antihipertensivos y a las guías basadas en la } \\
\text { evidencia para el comportamiento dietético y la } \\
\text { actividad física. }\end{array}$ & $\begin{array}{l}\text { Ensayo clínico aleatorizado. } \\
\text { Nivel de evidencia: } 1 c \text {. }\end{array}$ & 261 \\
\hline
\end{tabular}




\begin{tabular}{|c|c|c|c|c|}
\hline $\begin{array}{l}\text { Referencia } \\
\text { bibliográfica }\end{array}$ & Idioma, País & Objetivo & $\begin{array}{l}\text { Diseño/metodológico } \\
\text { Nivel de evidencia }\end{array}$ & Participantes \\
\hline $\begin{array}{l}\text { Vervloet et al., } \\
\text { (2014). }\end{array}$ & $\begin{array}{l}\text { Inglés. } \\
\text { Holanda. }\end{array}$ & $\begin{array}{l}\text { Investigar los efectos a corto y largo plazo del } \\
\text { uso de medicamentos de monitoreo en tiempo } \\
\text { real combinados con recordatorios del servicio de } \\
\text { mensajes cortos (SMS, por sus siglas en inglés) } \\
\text { para las dosis omitidas en la adherencia de relleno } \\
\text { a la medicación antidiabética oral. }\end{array}$ & $\begin{array}{l}\text { Ensayo clínico aleatorizado. } \\
\text { Nivel de evidencia: } 1 c \text {. }\end{array}$ & 110 \\
\hline $\begin{array}{l}\text { Esmatjes et } \\
\text { al., (2014). }\end{array}$ & $\begin{array}{l}\text { Inglés. } \\
\text { España. }\end{array}$ & $\begin{array}{l}\text { Evaluar el impacto de un sistema telemático } \\
\text { basado en Internet sobre el manejo económico y } \\
\text { clínico de pacientes con diabetes mellitus tipo } 1 .\end{array}$ & $\begin{array}{l}\text { Ensayo clínico aleatorizado. } \\
\text { Nivel de evidencia: } 1 c \text {. }\end{array}$ & 118 \\
\hline $\begin{array}{l}\text { McMahon et } \\
\text { al., (2012). }\end{array}$ & $\begin{array}{l}\text { Inglés. } \\
\text { Estados Unidos. }\end{array}$ & $\begin{array}{l}\text { Investigar si la administración de atención } \\
\text { telefónica o en línea mejora los resultados } \\
\text { relacionados con la diabetes a lo largo del } \\
\text { tiempo en comparación con la atención habitual } \\
\text { complementada con acceso a Internet y } \\
\text { capacitación. }\end{array}$ & $\begin{array}{l}\text { Ensayo clínico aleatorizado. } \\
\text { Nivel de evidencia: } 1 c \text {. }\end{array}$ & 118 \\
\hline $\begin{array}{l}\text { Noh et al., } \\
(2010) \text {. }\end{array}$ & $\begin{array}{l}\text { Inglés. } \\
\text { Corea. }\end{array}$ & $\begin{array}{l}\text { Evaluar el efecto de un sistema de información } \\
\text { completo basado en la web, que consiste en el uso } \\
\text { de Internet y de teléfonos celulares, en el control } \\
\text { de la glucosa en sangre. }\end{array}$ & $\begin{array}{l}\text { Ensayo clínico aleatorizado. } \\
\text { Nivel de evidencia: } 1 c \text {. }\end{array}$ & 40 \\
\hline $\begin{array}{l}\text { Shaya et al., } \\
\text { (2013). }\end{array}$ & $\begin{array}{l}\text { Inglés. } \\
\text { Estados Unidos. }\end{array}$ & $\begin{array}{l}\text { Evaluaron el efecto de la educación administrada } \\
\text { dentro de las redes sociales en la mejora de la } \\
\text { hipertensión en afroamericanos en comparación } \\
\text { con los controles históricos. }\end{array}$ & $\begin{array}{l}\text { Estudio cuasi-experimental. } \\
\text { Nivel de evidencia: } 2 \mathrm{a} \text {. }\end{array}$ & 441 \\
\hline $\begin{array}{l}\text { Supachaipani- } \\
\text { chpong et al., } \\
\text { (2018). }\end{array}$ & $\begin{array}{l}\text { Inglés. } \\
\text { Tailandia. }\end{array}$ & $\begin{array}{l}\text { Evaluar los efectos de una intervención de } \\
\text { educación sobre medicamentos integrada en los } \\
\text { servicios de rutina de una clínica de diabetes. }\end{array}$ & $\begin{array}{l}\text { Estudio cuasi-experimental. } \\
\text { Nivel de evidencia: } 2 \mathrm{a} \text {. }\end{array}$ & 76 \\
\hline $\begin{array}{l}\text { Kumar et al., } \\
\text { (2018). }\end{array}$ & $\begin{array}{l}\text { Inglés. } \\
\text { Estados Unidos. }\end{array}$ & $\begin{array}{l}\text { Evaluar el impacto de una aplicación móvil para } \\
\text { la diabetes con el asesoramiento en la aplicación } \\
\text { de un educador certificado en diabetes sobre el } \\
\text { control glucémico para personas con diabetes } \\
\text { tipo } 2 \text {. }\end{array}$ & $\begin{array}{l}\text { Estudio cuasi-experimental. } \\
\text { Nivel de evidencia: } 2 a \text {. }\end{array}$ & 292 \\
\hline $\begin{array}{l}\text { Walker et al., } \\
\text { (2017). }\end{array}$ & $\begin{array}{l}\text { Inglés. } \\
\text { Estados Unidos. }\end{array}$ & $\begin{array}{l}\text { Capturar la evidencia más reciente y relevante } \\
\text { relacionada con el uso de telemedicina en el } \\
\text { hogar como una intervención para los adultos } \\
\text { mayores con diabetes. }\end{array}$ & $\begin{array}{l}\text { Revisión Sistemática. } \\
\text { Nivel de evidencia: } 1 b .\end{array}$ & 14.370 \\
\hline $\begin{array}{l}\text { Liu et al., } \\
\text { (2013). }\end{array}$ & $\begin{array}{l}\text { Inglés. } \\
\text { Estados Unidos. }\end{array}$ & $\begin{array}{l}\text { Examinar la eficacia de las intervenciones } \\
\text { basadas en internet para reducir la presión } \\
\text { arterial sistólica y la presión arterial diastólica. }\end{array}$ & $\begin{array}{l}\text { Revisión Sistemática. } \\
\text { Nivel de evidencia: } 1 b .\end{array}$ & 2.221 \\
\hline $\begin{array}{l}\text { Farmer et al., } \\
\text { (2016). }\end{array}$ & $\begin{array}{l}\text { Inglés. } \\
\text { Estados Unidos. }\end{array}$ & $\begin{array}{l}\text { Evaluar el impacto de las intervenciones } \\
\text { que promueven el monitoreo del uso de los } \\
\text { medicamentos y los mensajes breves para apoyar } \\
\text { el cumplimiento de la medicación en pacientes } \\
\text { con diabetes mellitus tipo II, e investigar el } \\
\text { alcance del uso teórico para guiar el desarrollo de } \\
\text { la intervención. }\end{array}$ & $\begin{array}{l}\text { Revisión Sistemática. } \\
\text { Nivel de evidencia: la. }\end{array}$ & 4.820 \\
\hline
\end{tabular}

Fuente. Elaborada por los investigadores. 


\section{Categorías de análisis}

Al realizar en análisis de la información presentada se identificaron tres características asociadas a la efectividad del uso de las TICs en la adherencia terapéutica en personas con hipertensión arterial y diabetes meIlitus. Estas características fueron agrupadas por categorías de análisis descritas de la siguiente manera.

\section{Intervenciones Unicomponente}

En esta categoría se encontraron 13 estudios que utilizan una sola intervención empleadas en los pacientes que se encuentran inscritos en los programas de diabetes mellitus e hipertensión arterial mediante el uso de TICs con el objetivo de mejorar el acceso en atención oportuna en las consultas en salud y fortalecer el seguimiento a la adherencia al tratamiento prescrito, frente al cuidado que se brinda de manera habitual.

\section{Soporte telefónico}

Se encontraron 5 estudios que emplearon el uso de teléfonos como estrategia educativa, mostrando beneficios como el fortalecimiento del autocuidado y adherencia terapéutica. En el estudio de Supachaipanichpong et al. ${ }^{16}$, el soporte telefónico respalda el concepto de que la calidad y la comunicación efectiva de los proveedores de atención médica (tanto médicos como enfermeras) puede mejorar la comprensión de una persona de la medicación y la motivación para adherirse a esa medicación. Evidenciado en resultados estadísticamente significativos para el control de hemoglobina glicosilada, glicemia en ayuno, presión arterial, adherencia a medicamentos, conocimientos de los mismos, dieta y gasto energético 23,3,13,16.

Sin embargo, en los estudios de Canata et al. ${ }^{2}$, y Kanadli et al. ${ }^{9}$, no se encontraron diferencias estadísticamente significativas para variables como colesterol total, lipoproteína de baja densidad, lipoproteínas de alta densidad, triglicéridos, índice de masa corporal, circunferencia abdominal donde concluyen que no se produjo una reducción importante debido al corto tiempo de intervención.

\section{Programa en web}

En siete de los estudios que incluyeron programas basados en la web como herramienta válida con impacto positivo en la asistencia a los controles de salud y con similares resultados a los de una consulta habitual, según Avdal et al. ${ }^{10}$, el seguimiento basado en la web puede considerarse como una herramienta complementaria para el proceso de atención integral en personas con hipertensión arterial y diabetes mellitus ya que algunos pacientes presentan limitaciones a nivel laboral por su grado de ocupación y en otros casos limitaciones físicas que impiden el traslado a los servicios de salud.

En esta intervención se encontró significancia estadística para parámetros de hemoglobina glicosilada, tasa de asistencia médica, cifras tensionales, satisfacción terapéutica, costo-efectividad, adherencia medicamentosa, conocimiento del tratamiento, disminución en síntomas de hiperglicemia y mejores resultados en seguimiento con web a través de teléfonos móviles ${ }^{10,17-21}$. Para Esmatjes et al. ${ }^{17}$, el empleo de esta herramienta es ventajosa ya que es menos costosa, segura y efectiva. Según Liu et al. ${ }^{12}$, el uso de estos programas se ve favorecido por un tiempo mayor a 6 meses o más. Pese a estos hallazgos no hay significancia estadística para glicemia en ayunas, peso e índice de masa corporal ${ }^{20,21}$.

\section{Mensajería de texto}

Para el uso de mensajería de texto se incluyó 1 estudio. Para Vervloet et al. ${ }^{22}$, un sistema de recordatorio basado en mensajes de texto para la administración de medicamentos con el cual se monitorea el consumo de éstos en tiempo real permitió una mejora en la adherencia al tratamiento, con importancia estadística que a su vez fortaleció la autogestión de los pacientes, manteniendo estos resultados positivos a largo plazo.

\section{Intervenciones Multicomponente}

En esta categoría se encontraron 5 estudios que utilizaban intervenciones combinadas para lograr la adherencia al tratamiento.

\section{Programa en web y soporte telefónico}

Esta intervención abarcó 3 estudios que emplearon estrategias interactivas basadas en la tecnología vinculando a la administración del cuidado de enfermería, que en el estudio Glasgow et al. ${ }^{23}$, contribuyeron a mejorar las percepciones de los pacientes en su cuidado, calidad de vida y habilidades conductuales de autogestión. Al incorporar estas herramientas se evidenció resultados con valor significativo en cuanto a colesterol total, control de la angustia, hábitos alimenticios y actividad física; sin valor significativo en adherencia 
terapéutica, índice de masa corporal y lípidos, cabe resaltar que existen resultados contradictorios en los estudios incluidos en esta intervención para hemoglobina glicosilada y cifras tensionales ${ }^{23,24}$. Según Walker et al. ${ }^{25}$, la utilización de tecnología para educar, entrenar y monitorizar a distancia disminuye el deterioro cognitivo, la mortalidad, los ingresos hospitalarios y los costes de la atención médica; aumenta también el conocimiento, optimiza la adherencia y la autoeficacia.

\section{Programas en web y mensajería de texto}

El estudio proporcionado por Lee et al. ${ }^{26}$, manifiesta que la inclusión de estas intervenciones lograron generar cambios positivos para cifras tensionales y auto medición de tensión arterial, corroborando que al implementar un sistema basado en la nube para personas con hipertensión arterial y recordatorios de mensajes de texto genera beneficios para el control de la misma en comparación a la atención tradicional. Adicional a esto, el papel del personal de salud contribuye a un refuerzo positivo cuando emplea elogios por el buen comportamiento.

\section{Programas web, mensajería de texto y soporte te- lefónico}

En el estudio de Farmer et al. ${ }^{14}$, incluido para el análisis de la combinación de estas intervenciones presentó tamaños de efecto pequeños, con baja calidad metodológica, pero estadísticamente significativos, debido a esto sugiere investigaciones de alta calidad basadas en la teoría.

\section{Rol de enfermería en el uso de TICs para favorecer la adherencia terapéutica}

El rol de enfermería debe estar sujeto a contantes cambios e innovaciones como lo es la implementación del uso de herramientas de TICs dentro del campo de práctica que facilitan el acceso a las personas con patologías como diabetes mellitus e hipertensión arterial teniendo en cuenta que son las principales precursoras de le enfermedad renal crónica. Los profesionales de enfermería pueden apoyarse en las TICs implementando estrategias innovadoras tanto para la planeación como la ejecución de las actividades encaminadas a un cumplimiento satisfactorio del tratamiento, logrando un adecuado control metabólico, lo que repercute en una mejor calidad de vida ${ }^{2,9}$.

La adopción de estrategias innovadoras pueden implementarse en programas de salud pública, que implican beneficios de coste-efectividad y diversa adaptación cultural, así como fortalecimiento al acceso de consultas de atención en salud y favorece la reducción de tiempo empleado tanto para el paciente como para el personal de salud, a su vez el autocuidado en las personas con estas enfermedades mejora sustancialmente, debido a que adquieren información constante que optimiza sus conocimientos y autogestión de la enfermedad ${ }^{13,22}$.

En la Tabla 2 se presentan las intervenciones estadísticamente significativas, de acuerdo con los resultados encontrados.

\section{Discusión}

Con esta revisión sistemática se logró la síntesis de varios estudios que contenían el tema de interés acerca de la efectividad del uso de las TICs en la adherencia terapéutica en personas con diabetes mellitus e hipertensión arterial. Los estudios incluían estrategias de educación y seguimiento a través de portales web, mensajería de texto y llamadas telefónicas, con el fin de alcanzar los objetivos terapéuticos establecidos para el manejo de estas enfermedades que permiten implementar políticas, planes y programas de cuidado efectivos para la atención de este tipo de pacientes ${ }^{27}$. Los estudios incluidos son favorables en cuanto al grado de evidencia científica y fuerza de recomendación, encontrando que el $66,6 \%$ tuvo un nivel de evidencia 1 con recomendación c, el 16,6\% tuvo un nivel de evidencia 2 con recomendación a, el $11,1 \%$ tuvo un nivel de evidencia 1 con recomendación b y el 6,6\% tuvo un grado de evidencia 1 con recomendación a asignados según la propuesta del Instituto Joanna Briggs.

Glasgow et al.23, afirman que un aumento en la frecuencia de estrategias interactivas basadas en la tecnología vinculado a la administración de cuidado de enfermería puede generar efectos moderados tanto en el cuidado, calidad de vida y habilidades conductuales de autogestión de los pacientes, así como cambios de comportamiento, mejoras en los resultados biológicos y en aspectos psicosociales. En esta misma línea, Liu et al. ${ }^{12}$, manifiestan que el periodo de cada intervención tenía que ser de ocho semanas como mínimo para conseguir resultados clínicamente considerables, a su vez permitía la capacidad del participante de adquirir conocimientos y asumir nuevos comportamientos. Es por ello que se observó que las intervenciones basadas en Internet pueden ser más eficaces cuando un programa dura seis meses o más tiempo. Este hallazgo se confirma con los resultados de un estudio piloto realizado en Estados Unidos que midió el efecto de estrategias de 
Tabla 2. Intervenciones estadísticamente significativas.

\begin{tabular}{|c|c|c|c|c|}
\hline Intervención & & Autor & Variable & Valor $\mathbf{p}$ \\
\hline \multirow{12}{*}{$\begin{array}{l}\text { Intervenciones } \\
\text { unicomponente }\end{array}$} & \multirow{5}{*}{$\begin{array}{l}\text { Soporte } \\
\text { telefónico. }\end{array}$} & Kanadli et al., (2016). & Hemoglobina glicosilada. & $<0,001$ \\
\hline & & Canata et al., (2017). & Glicemia en ayuno. & $<0,0345$ \\
\hline & & Harrison et al., (2013). & Tensión arterial. & $<0,0001$ \\
\hline & & Supachaipanichpong et al., (2018). & Adherencia a medicamentos. & $<0,001$ \\
\hline & & Migneault et al., (2012). & Dieta. & $<0,03$ \\
\hline & \multirow{7}{*}{$\begin{array}{l}\text { Programas en } \\
\text { web. }\end{array}$} & \multirow{2}{*}{ Avdal et al., (2011). } & Hemoglobina glicosilada. & $<0,0001$ \\
\hline & & & Tasa de asistencia médica. & $<0,05$ \\
\hline & & Shaya et al., (2013). & Tensión arterial. & $<0,0001$ \\
\hline & & \multirow[t]{2}{*}{ Magid et al., (2013). } & Satisfacción terapéutica. & $<0,001$ \\
\hline & & & Adherencia a medicamentos. & $<0,001$ \\
\hline & & Esmatjes et al., (2014). & Costo-efectividad. & $<0,001$ \\
\hline & & Kumar et al., (2018). & Disminución de síntomas de hiperglicemia. & $<0,02$ \\
\hline \multirow{6}{*}{$\begin{array}{l}\text { Intervenciones } \\
\text { multicomponente }\end{array}$} & \multirow{4}{*}{$\begin{array}{l}\text { Programas en } \\
\text { web y soporte } \\
\text { telefónico. }\end{array}$} & \multirow{2}{*}{ McMahon et al., (2012). } & Colesterol total. & $<0,019$ \\
\hline & & & Control de angustia. & $<0,03$ \\
\hline & & \multirow{2}{*}{ Glasgow et al., (2012). } & Hábitos alimenticios. & $<0,05$ \\
\hline & & & Actividad física. & $<0,05$ \\
\hline & \multirow{2}{*}{$\begin{array}{l}\text { Programas en } \\
\text { web y mensajería } \\
\text { de texto. }\end{array}$} & \multirow[t]{2}{*}{ Lee et al., (2016). } & Tensión arterial. & $<0,0001$ \\
\hline & & & Auto-medición de tensión arterial. & $<0,0001$ \\
\hline
\end{tabular}

Fuente. Elaborada por los investigadores.

intervención por tres semanas consecutivas empleando mensajes cortos de texto el cual no arrojó cambios significativos para la variable evaluada ${ }^{28}$, lo cual respalda lo expuesto anteriormente.

En una revisión sistemática se encontró que otro de los factores relevantes en el proceso, fue el potencial de hacer que la atención en salud sea mejor, más rápida, menos costosa y más accesible al utilizar las tecnologías de la información y la comunicación ${ }^{29}$. En un estudio transversal realizado en Estados Unidos que incorporó video conferencia, red social en línea y chat grupal se evidenció el aumento en el alcance y el acceso a la atención, esto permitiría una disminución en costos especialmente para el paciente como para las entidades prestadoras de servicios de salud, lograría aumentar el acceso a la atención y reduciría la cantidad de tiempo y viajes que realiza el paciente ${ }^{30}$. A su vez en un estudio piloto realizado en Iraq demuestra que los mensajes SMS son aceptables, rentables y viables para respaldar el cuidado de la diabetes ${ }^{31}$. Esmatjes et al. ${ }^{17}$, evidenciaron que la aplicación de herramientas con uso de monitorización para las personas con diabetes mellitus e hipertensión arterial, es menos costosa y más eficiente que las visitas in situ.

Sin embargo, en una revisión sistemática se establecieron referencias valiosas con respecto al objetivo planteado: en un estudio expusieron que el uso de las TICs parece prometedor, aunque no concluyente. Sugirieron que la aplicación de las mismas debe evaluarse más directamente y que se necesitan métodos de estudio más rigurosos para mejorar la confiabilidad de los resultados ${ }^{32}$. Por otro lado, se encontró en una revisión sistemática un aporte que sugiere que los sistemas automatizados de comunicación telefónica pueden ser alentadores para cambiar las conductas en salud, mejorar los resultados de salud y aumentar el cuidado de la salud ${ }^{33}$.

Walker et al..$^{25}$, sugirió, "la extrema heterogeneidad de las intervenciones y los resultados evaluados, así como, en este caso, la escasez de estudios relevantes encontrados, hacen que sea difícil dibujar cualquier conclusión sólida, confiable o externamente válida de estos estudios como un todo". Por ende, es necesario una inves- 
tigación más intensa para entender qué intervenciones lograrían ser más efectivas en la población estudiada, e identificación de las necesidades y expectativas de esta población.

Farmer et al. ${ }^{14}$, refiere que, en cuanto a las intervenciones empleadas para optimizar la adherencia a la medicación, los hallazgos son limitados por ende es necesario investigación adicional de alta calidad fundamentada en la teoría. Una revisión sistemática de literatura realizada en Brasil deduce que el lograr una adherencia a medicamentos contribuye también a la evolución clínica favorable de la enfermedad y de la deserción de controles médicos en conjunto con la constante educación en cuanto a la enfermedad como al tratamiento. No obstante, la efectividad de este tipo de intervención deberá ser probada en estudios futuros a largo plazo ${ }^{8}$.

En cuanto a las implicaciones para la práctica de enfermería según Canata et al. ${ }^{2}$, las intervenciones por soporte telefónico y acompañamiento por los profesionales de enfermería puede agregar valor a las estrategias empleadas por el enfermero en la educación e incrementar la atención en los servicios de salud. En un estudio piloto realizado en Estados Unidos basado en programas de mensajes de texto, se evidenció mayor adherencia al tratamiento para el manejo de la diabetes y también se reforzó la importancia de la autogestión lo cual es de gran ayuda en el rol del enfermero ${ }^{34}$.

En un estudio cuasi-experimental realizado en Irán sugiere que el seguimiento regular por parte de un enfermero empleando tecnologías de la información y comunicación puede ayudar a monitorear el progreso del paciente y reforzar los comportamientos de salud relacionados con el cuidado de su enfermedad ${ }^{35}$.

Una limitación de esta revisión sistemática, es la diversidad de tipos de estudio incluidos para el proceso de análisis, aumentando el riesgo de sesgo para los resultados obtenidos en el mismo.

\section{Conclusión}

En el cuidado de la salud actualmente hay hallazgos iniciales que apoyan la efectividad del uso de las TICs, convirtiéndolas en una herramienta que favorece la adherencia al tratamiento en pacientes con hipertensión arterial y diabetes mellitus, estas estrategias presentan mejores resultados cuando el tiempo de la intervención es mayor a un año, de la misma manera logra reducir costos e inversión de tiempo tanto en el personal de salud, como en el paciente. El uso de estas TICs contribuye a los programas liderados por profesionales de enfermería como instrumentos innovadores, que pueden complementar las actividades de educación en salud y mejorar el seguimiento. Son necesarias investigaciones a futuro para comprender mejor qué intervenciones pueden ser más efectivas en esta población.

Recibido: 08-06-19

Revisado: 15-07-19

Modificado: 25-10-19

Aceptado: 15-12-19

\section{Bibliografía}

1. Ajay VS, Jindal D, Roy A, Venugopal V, Sharma R, Pawar A, et al. Development of a Smartphone-Enabled Hypertension and Diabetes Mellitus Management Package to Facilitate Evidence-Based Care Delivery in Primary Healthcare Facilities in India: The mPower Heart Project. J Am Heart Assoc. 2016;5(12):e004343.

2. Canata Becker TA, de Souza Teixeira CR, Zanetti $M L$, Pace $A E$, raújo Almeida $F A$, da Costa Gonçalves Torquato MT. Efeitos do suporte telefônico no controle metabólico de idosos com diabetes mellitus. Rev Bras Enferm. 2017;70(4):737-43.

3. Harrison TN, Ho TS, Handler J, Kanter MH, Goldberg RA, Reynolds K. A Randomized Controlled Trial of an Automated Telephone Intervention to Improve Blood Pressure Control. J Clin Hypertens. 2013;15(9):650-4.

4. World Health Organization. World health statistics 2007. Geneve: World Health Organization; 2007.

5. Ministerio de Salud y Protección Social. Análisis de situación de salud (ASIS) Colombia. Dirección de Epidemiología y Demografía. Bogotá, noviembre de 2016. 
6. Marzo-Castillejo M, Vela-Vallespín $C$, Bellas-Beceiro $B$, Bartolomé-Moreno $C$, Melús-Palazón E, Vilarrubí-Estrella $\mathrm{M}$ et al. Recomendaciones de prevención del cáncer. Actualización PAPPS 2018. Aten Primaria. 2018;50(Supl 1):S41-65.

7. Bloom BS. Daily regimen and compliance with treatment. BMJ. 2001;323(7314):647.

8. Prado CS, Tenório JM, Ruiz EES, Ortolani CLF, Pisa IT. Impacto da utilização de mensagens do tipo SMS (Short Message Service) como lembrete na adesão ao tratamento de saúde: revisão sistemática da literatura. J Heal Inf. 2012;4(4):159-64.

9. Kanadli KA, Ovayolu N, Ovayolu Ö. Does telephone follow-up and education affect self-care and metabolic control in diabetic patients? Holist Nurs Pract. 2016;30(2):70-7.

10. Avdal EÜ, Kizilci S, Demirel N. The effects of web-based diabetes education on diabetes care results: A randomized control study. CIN - Comput Informatics Nurs. 2011;29(2):101-6.

11. Belloch C. Las Tecnologías de la Información y Comunicación en el Aprendizaje. Unidad Tecnol Educ (UTE)- Dep Metod Investig y diagnostico en educación. 2012;1-9.

12. Liu S, Dunford SD, Leung YW, Brooks D, Thomas $S G$, Eysenbach $G$, et al. Reducing blood pressure with internet-based interventions: A meta-analysis. Can J Cardiol. 2013;29(5):613-21.

13. Migneault JP, Dedier JJ, Wright JA, Heeren T, Campbell MK, Morisky DE, et al. A culturally adapted telecommunication system to improve physical activity, diet quality, and medication adherence among hypertensive african-americans: A randomized controlled trial. Ann Behav Med. 2012;43(1):62-73.

14. Farmer AJ, Mcsharry J, Rowbotham S, Mcgowan L, Ricci-Cabello I, French DP. Effects of interventions promoting monitoring of medication use and brief messaging on medication adherence for people with Type 2 diabetes: A systematic review of randomized trials. Diabet Med. 2016;33(5):565-79.

15. Guirao Goris SJA. Utilidad y tipos de revisión de literatura. Ene. [Internet]. 2015 [Consultado 5 oct 2018]; 9(2). Disponible en: http:// scielo.isciii.es/scielo.php?script=sci_arttext\&pi$d=$ S1988-348X2015000200002.

16. Supachaipanichpong $P$, Vatanasomboon $P$, Tansakul $S$, Chumchuen P. An Education Intervention for Medication Adherence in Uncontrolled Diabetes in Thailand. PRIJNR 2018;22(2):144-56.

17. Esmatjes $E$, Jansà $M$, Roca $D$, Pérez-Ferre $N$, del VaIle L, Martínez-Hervás S, et al. The Efficiency of Telemedicine to Optimize Metabolic Control in Patients with Type 1 Diabetes Mellitus: Telemed Study. Diabetes Technol Ther [Internet]. 2014;16(7):435-41.

18. Shaya FT, Chirikov V V., Daniel Mullins C, Shematek J, Howard D, Foster C, et al. Social Networks Help Control Hypertension. J Clin Hypertens. 2013;15(1):34-40.

19. Magid DJ, Olson KL, Billups SJ, Wagner NM, Lyons EE, Kroner BA. A pharmacist-led, American Heart Association Heart360 Web-enabled home blood pressure monitoring program. Circ Cardiovasc Qual Outcomes. 2013;6(2):157-63.

20. Noh J-H, Cho Y-J, Nam H-W, Kim J-H, Kim D-J, Yoo $\mathrm{H}-\mathrm{S}$, et al. Web-Based Comprehensive Information System for Self-Management of Diabetes Mellitus. Diabetes Technol Ther. 2010;12(5):333-7.

21. Kumar S, Moseson H, Uppal J, Juusola JL. A Diabetes Mobile App With In-App Coaching From a Certified Diabetes Educator Reduces AlC for Individuals With Type 2 Diabetes. Diabetes Educ. 2018;44(3):226-36.

22. Vervloet $M$, van Dijk $L$, de Bakker $D H$, Souverein PC, Santen-Reestman J, van Vlijmen B, et al. Shortand long-term effects of real-time medication monitoring with short message service (SMS) reminders for missed doses on the refill adherence of people with Type 2 diabetes: Evidence from a randomized controlled trial. Diabet Med. 2014;31(7):821-8.

23. Glasgow RE, Kurz D, King D, Dickman JM, Faber AJ, Halterman $E$, et al. Twelve-month outcomes of an Internet-based diabetes self-management support program. Patient Educ Couns. 2012;87(1):81-92.

24. McMahon GT, Fonda SJ, Gomes HE, Alexis G, Conlin PR. A Randomized Comparison of Online- and Telephone-Based Care Management with Internet 
Training Alone in Adult Patients with Poorly Controlled Type 2 Diabetes. Diabetes Technol Ther. 2012;14(11):1060-7.

25. Walker CL, Kopp M, Binford RM, Bowers CJ. Home telehealth interventions for older adults with diabetes. Home Healthc Now. 2017 Apr;35(4):202-10.

26. Lee $P$, Liu JC, Hsieh MH, Hao WR, Tseng YT, Liu $\mathrm{SH}$, et al. Cloud-based BP system integrated with CPOE improves self-management of the hypertensive patients: A randomized controlled trial. Comput Methods Programs Biomed. 2016;132(2016):10513.

27. Stella M, Aldana C De, Plata DM. Chronic Non-Communicable Diseases and Use of Information. Rev Cuid. 2014;5(1):661-9.

28. Arora S, Peters AL, Agy C, Menchine M. A Mobile Health Intervention for Inner City Patients with Poorly Controlled Diabetes: Proof-of-Concept of the TExT-MED Program. Diabetes Technol Ther. 2012;14(6):492-6.

29. Gandapur Y, Kianoush S, Kelli HM, Misra S, Urrea $\mathrm{B}$, Blaha MJ, et al. The role of $\mathrm{mHealth}$ for improving medication adherence in patients with cardiovascular disease: A systematic review. Eur Hear J - Qual Care Clin Outcomes. 2016;2(4):237-44.

30. Shaw RJ, Ferranti J. Patient-provider internet portals - Patient outcomes and use. CIN - Comput Informatics Nurs. 2011;29(12):714-8.
31. Haddad NS, Istepanian R, Philip N, Khazaal FAK, Hamdan TA, Pickles T, et al. a feasibility study of mobile phone text messaging to support education and management of type 2 diabetes in Iraq. Diabetes Technol Ther. 2014;16(7):454-9.

32. Peterson A. Improving type 1 diabetes management with mobile tools: A systematic review. J Diabetes Sci Technol. 2014;8(4):859-64.

33. Posadzki P, Mastellos N, Ryan R, Lh G, Lm F, Pappas $Y$, et al. Automated telephone communication systems for preventive healthcare and management of long-term conditions. Cochrane Database Syst Rev. 2016 Dec 14;12:CD009921.

34. Nundy S, Dick J, Solomon M, Peek M.Developing a Behavioral Model for Mobile Phone-Based Diabetes Interventions. Patient Educ Couns. 2013 Jan;90(1):125-32.

35. Zolfaghari M, Mousavifar SA, Pedram S, Haghani $H$. The impact of nurse short message services and telephone follow-ups on diabetic adherence: Which one is more effective? J Clin Nurs. 2012;21(1314):1922-31.

Este artículo se distribuye bajo una Licencia Creative Commons Atribución-NoComercial 4.0 Internacional. https://creativecommons.org/licenses/by-nc/4.0/ 